\title{
Noninvasive screening identifies patients at risk for spontaneous bacterial peritonitis caused by multidrug-resistant organisms
}

This article was published in the following Dove Press journal: Infection and Drug Resistance

\author{
Philip G Ferstl ${ }^{1,2}$ \\ Mona Müller' \\ Natalie Filmann ${ }^{3}$ \\ Michael Hogardt ${ }^{2,4,5}$ \\ Volkhard AJ Kempf $f^{2,4,5}$ \\ Thomas A Wichelhaus ${ }^{2,4,5}$ \\ Christian M Lange ${ }^{1,2}$ \\ Johannes Vermehren ${ }^{1,2}$ \\ Stefan Zeuzem ${ }^{1,2}$ \\ Claudia Reinheimer $2,4,5$ \\ Oliver Waidmann ${ }^{1,2}$
}

'Department for Internal Medicine I/ Gastroenterology and Hepatology,

University Hospital Frankfurt,

Frankfurt am Main, Germany;

${ }^{2}$ University Center for Infectious

Diseases (UCI), University Hospital

Frankfurt, Frankfurt am Main,

Germany; ${ }^{3}$ Institute of Biostatistics and Mathematical Modeling, University Hospital Frankfurt, Frankfurt am

Main, Germany; ${ }^{4}$ Institute of Medical Microbiology and Infection Control, University Hospital Frankfurt, Frankfurt am Main, Germany;

5University Center of Competence for Infection Control at the

Universities Frankfurt, Giessen, and Marburg, Frankfurt am Main, State of Hesse, Germany

Correspondence: Philip G Ferst Department for Internal Medicine I/ Gastroenterology and Hepatology, University Hospital Frankfurt, TheodorStern-Kai 7, 60590 Frankfurt am Main,

Germany

Tel +4969630I 5I22

Email philip.ferstl@kgu.de
Background and aims: Spontaneous bacterial peritonitis (SBP) is a severe complication of decompensated cirrhosis. The prevalence of multidrug-resistant organisms (MDROs) in patients with cirrhosis is increasing. Identification of patients at risk for SBP due to MDROs (ie, SBP with the evidence of MDROs or Stenotrophomonas maltophilia in ascitic culture, MDRO-SBP) is crucial to the early adaptation of antibiotic treatment in such patients. We therefore investigated whether MDROs found in ascitic cultures can also be found in specimens determined by noninvasive screening procedures.

Patients and methods: This retrospective study was conducted at the liver center of the University Hospital Frankfurt, Germany. Between 2011 and 2016, patients with cirrhosis were included upon diagnosis of SBP and sample collection of aerobic/anaerobic ascitic cultures. Furthermore, the performance of at least one complete MDRO screening was mandatory for study inclusion.

Results: Of 133 patients diagnosed with SBP, 75 (56.4\%) had culture-positive SBP and 22 (16.5\%) had MDRO-SBP. Multidrug-resistant Escherichia coli (10/22; 45.5\%) and vancomycinresistant enterococci $(7 / 22 ; 36.4 \%)$ resembled the major causatives of MDRO-SBP. Rectal swabs identified MDROs in 17 of 22 patients (77.3\%) who developed MDRO-SBP with a time-dependent sensitivity of $77 \%$ and $87 \%$ after 30 and 90 days upon testing, while negative predictive value was $83 \%$ and $76 \%$, respectively. The majority of patients were included from intensive care unit or intermediate care unit.

Conclusion: MDRO screening may serve as a noninvasive diagnostic tool to identify patients at risk for MDRO-SBP. Patients with decompensated cirrhosis should be screened for MDROs from the first day of inpatient treatment onward.

Keywords: multidrug resistance, liver cirrhosis, ascites, screening routine, antibiotic therapy

\section{Plain language summary}

Patients with liver cirrhosis are prone to develop bacterial infections, which mitigate quality of life and survival. The occurrence of multidrug-resistant organisms (MDROs) puts these patients at additional risk and is therefore a severe life event. Since repetitive antibiotic therapies promote the occurrence of MDROs due to further selection pressure, the antibiotic regimen needs to be chosen carefully based on individual patient data. Therefore, the identification of patients at risk for infections caused by MDROs is vital. MDROs are mostly harbored in the large intestine or on the skin and can easily be diagnosed using screening swabs.

Spontaneous bacterial peritonitis (SBP) is the second most frequent bacterial infection in patients with cirrhosis and ascites and is caused by intestinal bacteria migrating from the gut to the abdomen. Due to the late onset of symptoms, diagnosis may be delayed, and special attention 
is warranted in the care of these patients. So far, studies determining the outcome of SBP caused by MDROs are scarce. We retrospectively compiled a cohort of patients with SBP who underwent at least one complete MDRO screening (rectal, nasopharyngeal and cutaneous). In this study, we described the clinical impact of ascitic MDRO detection and aimed to assess the utility of such noninvasive screening to identify patients at risk for SBP caused by MDROs.

\section{Introduction}

The prevalence of multidrug-resistant organisms (MDROs) in patients with cirrhosis is rapidly increasing. ${ }^{1,2}$ Bacterial infections have a devastating clinical impact in patients with decompensated cirrhosis, including prolonged and repeated hospitalization as well as reduced survival. ${ }^{3-5}$ Spontaneous bacterial peritonitis (SBP) is the second most common bacterial infection in patients with cirrhosis and ascites. ${ }^{6}$ Studies from different continents have determined cirrhotic patients' outcome as particularly poor in the case of infection by MDROs. . $, 3,7-13^{-13}$

MDRO-mediated infections in cirrhosis have mostly been attributed to multidrug-resistant gram-negative bacteria (MDRGN). ${ }^{14,15}$ Secondary SBP prophylaxis with fluoroquinolones has been recommended for more than 2 decades, but multidrug resistance narrows therapeutic options. ${ }^{15-18}$ In addition, non-fermenting gram-negative bacteria expressing multidrug resistance, such as Stenotrophomonas maltophilia, might bloom under recurrent administration of antimicrobials. ${ }^{4,1920}$ In addition, gram-positive pathogens such as enterococci are increasingly contributing to the poor prognosis of SBP, since patients with cirrhosis are at high risk to acquire vancomycin-resistant enterococci (VRE). ${ }^{15,21-23}$ The burden and spread of VRE are associated with extensive and repetitive antibiotic therapies. ${ }^{24,25}$ The occurrence of these pathogens is being promoted by intestinal dysbiosis, putting patients with cirrhosis at high risk for acquiring SBP by MDROs (ie, SBP with the evidence of MDROs or S. maltophilia in ascitic culture, MDRO-SBP). ${ }^{19,20}$

Among the entities of bacterial infection in cirrhosis, SBP is particularly harmful due to the late onset of symptoms, risk of recurrence and the wide spectrum of causative bacteria. ${ }^{4,10,15,21,22,26}$ To guarantee optimal patient care and, at the same time, minimize selective pressure on further MDRO development, an individualized antibiotic regimen based on concise patient-specific data may be warranted. Therefore, the anticipation of expectable pathogens would be vital. Since SBP is derived from the intestinal microflora, it seems feasible to investigate whether these pathogens may be detected earlier by noninvasive procedures. In this retrospective study, we assessed the diagnostic utility of rectal, nasopharyngeal and cutaneous MDRO screening in a cohort of patients with decompensated cirrhosis, and we described the microbiological characteristics and clinical impact of MDRO-SBP in a tertiary liver transplant center in Germany.

\section{Patients and methods Study characterization}

This study was conducted at the liver transplant center at University Hospital Frankfurt (UHF), Germany. From an electronic database, we retrieved all cases coded as "acute peritonitis" (ICD codes such as K65.0, K65.8 and K65.9) from 2012 to 2016 . We retrieved 419 cases in total, which are represented by 299 individuals receiving inpatient treatment in the given period. Inclusion criteria were as follows: 1) diagnosis of SBP as defined by polymorphonuclear leukocyte count of $>250 / \mu \mathrm{L}$ in ascites in patients with cirrhosis. Patients were included upon the first SBP episode diagnosed at UHF. 2) Sample collection of at least one pair of aerobic/anaerobic ascitic cultures at the bedside at the time of diagnosis of SBP. 3) Complete MDRO screening (rectal, throat, skin, nose swabs). Exclusion criteria were as follows: patients with primary peritonitis or malignant ascites due to any abdominal metastases or peritoneal carcinosis. Biometric, clinical, laboratory and microbiological data as well as empiric antibiotic therapy were collected at the date of study inclusion. If more detailed patient characterization was deemed necessary, the corresponding data were retrieved at defined time points after study inclusion. All the patient data were retrieved from electronic patient charts. Importantly, further microbiological data were collected from study inclusion until death or lost to follow up, resulting in multiple microbiological test results for most patients.

\section{Colonization, infection and case definition}

If MDROs were detected only in rectal, throat or cutaneous swabs, patients were defined as "colonized". If the MDRO strain was detected only or additionally in ascites, blood, urine, bronchial or pleural secretion, bile, pus, wounds or surgical sites, on medical devices or catheters, the patient was classified as "infected" by the strain. "Nosocomial SBP" was defined as SBP diagnosed at least 3 days after hospitalization according to the current definition ${ }^{27,28}$ or as SBP acquired up to 7 days after the last hospital discharge. Sepsis was defined as suspected or evident bacterial infection combined with two of the following clinical parameters according to the quick sequential organ failure assessment (qSOFA) score ${ }^{29}$ : 
hemodynamic instability with systolic blood pressure below $100 \mathrm{mmHg}$ (including patients receiving noradrenaline with systolic blood pressure $>100 \mathrm{mmHg}$ ), respiratory rate $>22$ / min or $\mathrm{PaCO}_{2}<32 \mathrm{mmHg}$ and/or altered mental status.

\section{Screening for MDROs at UHF}

Screening routine was performed for MDRGN, VRE and methicillin-resistant Streptococcus aureus (MRSA). No regular screening was scheduled for S. maltophilia. MDRO screening was performed according to the infection control protocol at UHF which again is according to the German infection protection law ( $\$ 23$, Infektionsschutzgesetz $\left.{ }^{30}\right)$. Screening measures apply to, eg, patients arriving from hospitals in high prevalence countries/high prevalence local hospitals for MDRGN and/or MRSA as well as patients admitted to intensive care unit/intermediate care unit (ICU/ IMC) on the day of admission. In addition, MDRO screening is scheduled weekly on ICU and IMC at UHF, and patients included may therefore have undergone repetitive MDRO screening. As of 2011-2016, no regular MDRO screening was scheduled on normal hepatologic wards.

\section{Definition of MDRO-SBP}

MDRO-SBP was defined as SBP due to MDRGN, VRE and MRSA as well as due to $S$. maltophilia. Although $S$. maltophilia intrinsically exhibits a broad spectrum of antibiotic resistance, eg, against antibiotic resistance, eg against carbapenems, it does not genuinely qualify to be included in the collective term “MDRO”. However, S. maltophilia, MDRGN, VRE and MRSA all require treatment with broad-spectrum antibiotics. We therefore decided to include $S$. maltophilia in the collective term "MDRO-SBP".

\section{Definition of MDRGN}

MDRGN were defined as Enterobacteriaceae with extendedspectrum ß-lactamase (ESBL) phenotype as well as Enterobacteriaceae, Pseudomonas aeruginosa and Acinetobacter baumannii resistant against piperacillin, any third- or fourthgeneration cephalosporin and fluoroquinolone resistance $(\mathrm{QR}) \pm \mathrm{CR} \cdot{ }^{31,32}$

\section{Detection of MDROs and molecular resistance analysis}

All laboratory procedures were performed under qualitycontrolled criteria (laboratory accreditation according to ISO 15189:2007 standards; certificate number D-ML-13102-0100 , valid through January 25, 2021). To identify MDRGN and VRE, rectal swabs were collected using culture swabs with
Amies collection and transport medium (Hain Lifescience, Nehren, Germany) and streaked onto selective CHROMagar ESBL plates (Mast Diagnostica, Paris, France) and ChromID VRE agar (bioMérieux, Nürtingen, Germany), respectively. Species identification was performed by matrix-assisted laser desorption ionization-time-of-flight analysis (VITEK MS; bioMérieux). Antibiotic susceptibility testing was performed according to Clinical and Laboratory Standards Institute guidelines using VITEK 2 and/or antibiotic gradient tests (bioMérieux). In the case of gram-negative CR isolates, the detection of genes encoding carbapenemases was routinely performed via PCR analysis and subsequent sequencing including the bla genes for carbapenemases NDM, VIM, IMP, OXA-48 like, and KPC as well as OXA-23, OXA-24 and OXA-58 for Acinetobacter baumannii. ${ }^{33,34}$ For the detection of MRSA, moistened nasal swabs were inoculated on Brilliance MRSA Agar (Oxoid, Wesel, Germany), and identification and antimicrobial susceptibility testing were performed as described earlier. The detection of MDROs from screening and microbiological culture of clinical specimens (eg, ascites) were performed according to German microbiological procedure quality standards (MiQ), for example, current versions such as MiQ 2, 3a/b (2007), MiQ 25 (2006), MiQ 32 (2012).

\section{Ethics statement}

For this retrospective study, approval from the clinical ethics committee of the Goethe University Frankfurt was obtained prior to research (file number: 391/15). The database was anonymized, and informed consent was waived due to the retrospective nature of the study.

\section{Statistical analysis}

BiAS software (v. 11.06; Epsilon-Verlag, Darmstadt, Germany) and R (v. 3.2.0, R Core Team [2015], R: A language and environment for statistical computing; R Foundation for Statistical Computing, Vienna, Austria) were used for statistical calculations. Fatal outcome due to sepsis, fatal outcome due to other causes and the appearance of MDRO-SBP were defined as end points, and patients who did not reach an end point were censored from the last day of inpatient treatment. Model for End-stage Liver Disease (MELD), AlbuminBilirubin (ALBI), Child-Pugh-Turcotte and Chronic Liver Failure-Acute Decompensation (CLIF-AD) scores were calculated with the laboratory parameters taken at the day of SBP detection. Standard deviation (SD) was calculated for age, MELD and CLIF-AD scores and laboratory parameters. $\mathrm{SE}$ of the mean was calculated for all observational intervals given in days. Clinical scores were compared using the 
Wilcoxon-Mann-Whitney $U$-test. Kaplan-Maier curve was employed for the display of comparative survival analysis, and Cox-Mantel chi-squared test was used for the calculation of HR and $P$-values. Considering the appearance of MDROSBP as the event of interest, fatal outcome due to sepsis and fatal outcome due to other causes than sepsis were defined as competing risks, using the Aalen-Johansen estimator. In case that death fatal due to sepsis was considered as the event of interest, fatal outcome due to other causes than sepsis was defined as competing risk. Independent-risk factors for death were calculated using the multivariate Cox regression model. For calculating unbiased sensitivity and specificity for censored data with competing risks, the method proposed by Blanche et $\mathrm{al}^{35}$ was utilized.

\section{Results}

\section{Clinical characterization of the cohort}

One hundred and thirty-three patients diagnosed with SBP between December 2011 and August 2016 were included in the analysis (Figure S1). Of these, 72 (54.1\%) were positive for MDRO colonization or infection in any location, while MDRO-SBP was diagnosed in 22 patients (16.5\%). Biometric and laboratory parameters as well as liver scores (ChildPugh-Turcotte, MELD, CLIF-AD, ALBI) did not differ between patients with or without the detection of MDROs or MDRO-SBP, respectively. Clinical features of hepatic decompensation were equally distributed (Table 1). MELD score and ICU admission at the time of SBP diagnosis were independent predictors of survival, and MELD, CLIF-AD and ALBI scores were higher in patients who died than those in patients who were lost to follow up (Table S1). Patients with MDRO-SBP were at increased risk of death $(\mathrm{HR}=2.17$, $P=0.007)$ and were more likely to develop lethal sepsis (HR $=5.67, P<0.001)$. While MELD and CLIF-AD scores were marginally better in patients with culture-negative SBP, severe clinical courses due to bacterial infections were more frequent in patients with MDRO-SBP (Figure 1). As calculated by multivariate regression, liver scores or clinical features were not associated with the occurrence of MDRO-SBP.

\section{Microbiological characterization of the cohort}

The first MDRO screening was performed before or upon study inclusion in $105 / 133$ patients $(78.9 \%)$ and in timely fashion in the rest of the patients, resulting in MDRO detection in the majority of patients (Figure 2A and B). Among $72 / 133$ patients with MDROs, MDRGN were detected in $42 / 133$ cases $(31.6 \%)$, VRE in $50 / 133$ cases $(37.6 \%)$ and MRSA in $5 / 133$ cases $(3.8 \%)$. Colonization - defined as positivity for MDRO screening without detection in invasive materials - was detected in $16 / 42$ patients $(38.1 \%)$ with MDRGN, 39/50 patients (78\%) with VRE and 2/5 patients $(40 \%)$ with MRSA. MDRO-associated ascitic or extra-ascitic infection was found in the remaining patients within the body compartments as given in Table 2. Multiple MDRO strains were detected within 24 individuals, resulting

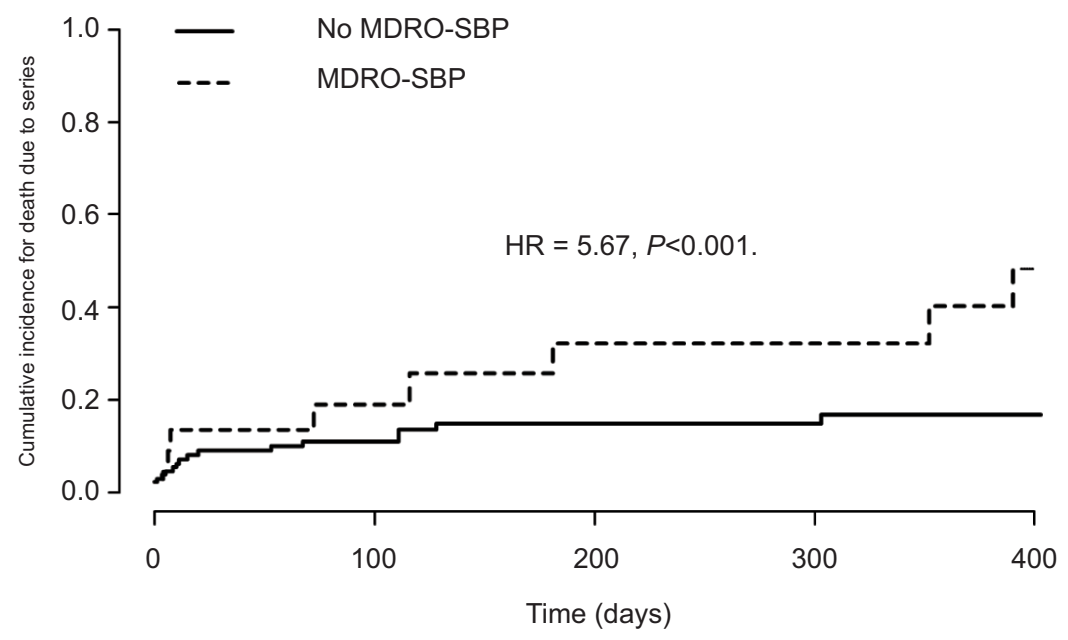

Figure I Competing risk analysis of 22 patients with MDRO-SBP compared to 11 I patients without MDRO-SBP.

Notes: Patients were included from the day of first SBP detection, and death due to sepsis and death due to other reasons were defined as competing risks. Lethal sepsis was significantly increased in patients with MDRO-SBP $(H R=5.67, P<0.001)$. Of note, MDRO-SBP was included as a time-dependent variable in this analysis. Therefore, the number at risk cannot be displayed in this plot, since the time-dependent calculation has been performed with censored data. ${ }^{35}$ MDRO-SBP, SBP with the evidence of MDROs or S. maltophilia in ascitic culture.

Abbreviations: MDRO, multidrug-resistant organism; SBP, spontaneous bacterial peritonitis; S. maltophilia, Stenotrophomonas maltophilia. 
Table I General characteristics of the included patients

\begin{tabular}{|c|c|c|c|c|c|}
\hline \multicolumn{2}{|c|}{ 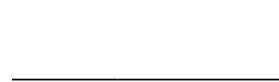 } & All patients & $\begin{array}{l}\text { Patients with } \\
\text { MDROs }\end{array}$ & $\begin{array}{l}\text { Patients with } \\
\text { MDRO-SBP }\end{array}$ & $\begin{array}{l}\text { Patients with } \\
\text { culture-negative SBP }\end{array}$ \\
\hline \multicolumn{2}{|c|}{$\sum$} & 133 (100\%) & $72(54.1 \%)$ & $22(16.5 \%)$ & $58(43.6 \%)$ \\
\hline \multicolumn{6}{|c|}{$\begin{array}{l}\text { Biometric } \\
\text { characteristics }\end{array}$} \\
\hline \multicolumn{2}{|c|}{$\begin{array}{l}\text { Age (year), mean } \pm S D \\
\text { (range) }\end{array}$} & $55.1 \pm 11.2(28-86)$ & $56.1 \pm 10.8(28-74)$ & $54.1 \pm I I .9(46-74)$ & $57.1 \pm 11.6(30-86)$ \\
\hline \multicolumn{2}{|c|}{ Male sex } & 98 (73.7\%) & $53(73.6 \%)$ & 20 (90.9\%) & $37(63.8 \%)^{\mathrm{a}}$ \\
\hline \multicolumn{2}{|c|}{$\begin{array}{l}\text { Mean observational } \\
\text { time (d), mean (range) }\end{array}$} & $230(I-I, 529)$ & $206(I-1,109)$ & $174(I-799)$ & $264(I-I, 529)$ \\
\hline \multicolumn{6}{|c|}{ Liver scores } \\
\hline \multicolumn{2}{|c|}{ MELD } & $22.4(7-40)$ & $23.1(11-40)$ & $24.4(13-40)$ & $20.3(7-40)^{\mathrm{a}}$ \\
\hline \multicolumn{2}{|c|}{ Child A } & $\mathrm{I}(0.8 \%)$ & I (I.4\%) & $0(0 \%)$ & I (I.7\%) \\
\hline \multicolumn{2}{|c|}{ Child B } & 35 (26.3\%) & 14 (19.4\%) & $3(13.6 \%)$ & $21(36.2 \%)$ \\
\hline \multicolumn{2}{|c|}{ Child C } & 97 (72.9\%) & 57 (79.2\%) & $19(86.4 \%)$ & $36(62.1 \%)$ \\
\hline \multicolumn{2}{|c|}{ CLIF-AD } & $61.9(32-89)$ & $63.3(43-88)$ & $64.4(43-88)$ & $59.2(32-85)^{\mathrm{a}}$ \\
\hline \multicolumn{2}{|c|}{ ALBI } & $1.15(0.35-1.9)$ & $1.17(0.55-1.89)$ & $1.21(0.55-1.65)$ & $1.09(0.35-1.9)$ \\
\hline \multicolumn{6}{|c|}{$\begin{array}{l}\text { Etiology of } \\
\text { cirrhosis }^{b}\end{array}$} \\
\hline \multicolumn{2}{|c|}{ Alcoholic } & $80(60.2 \%)$ & 44 (61.1\%) & $15(68.2 \%)$ & 37 (63.8\%) \\
\hline \multicolumn{2}{|c|}{$\mathrm{HBV} / \mathrm{HCV}$} & 45 (33.8\%) & 23 (31.9\%) & 7 (31.8\%) & $18(31 \%)$ \\
\hline \multicolumn{2}{|c|}{ Others ${ }^{c}$} & $19(14.3 \%)$ & II (I5.3\%) & $4(18.2 \%)$ & $10(17.2 \%)$ \\
\hline \multicolumn{6}{|c|}{ Laboratory } \\
\hline \multicolumn{2}{|c|}{ Sodium (mmol/L) } & I34.I (II0-159) & |33.| (||8-149) & $|3| .5(|| 8-\mid 45)^{\mathrm{a}}$ & $136.2(122-159)^{\mathrm{a}}$ \\
\hline \multicolumn{2}{|c|}{ Creatinine (mg/dL) } & $2.01(0.45-7.66)$ & $2.08(0.60-7.66)$ & $2.34(0.60-7.66)$ & $\mathrm{I} .83(0.45-4.6 \mathrm{I})$ \\
\hline \multicolumn{2}{|c|}{ Bilirubin (mg/dL) } & $6.4(0.2-44.6)$ & $6.4(0.4-43.1)$ & $5.9(0.4-18.6)$ & $6.1(0.2-44.6)$ \\
\hline \multicolumn{2}{|c|}{ Albumin (g/dL) } & $2.8(1.5-4.4)$ & $2.8(1.5-4.3)$ & $2.7(1.5-4)$ & $2.9(1.8-4.4)$ \\
\hline \multicolumn{2}{|c|}{ INR } & $1.73(1.04-3.75)$ & $1.77(1.04-3.1)$ & $1.86(1.04-3.1)$ & $1.59(1.05-2.79)^{\mathrm{a}}$ \\
\hline \multicolumn{2}{|c|}{ 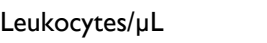 } & $11.6(2-96.1)$ & $12.6(2.7-96.1)$ & $11.8(3.1-23.8)$ & $12.7(2.4-96.1)$ \\
\hline $\operatorname{CRP}(\mathrm{n}$ & $g / d L)$ & $7.1(0.2-30.4)$ & $7.4(0.2-30.4)$ & $6.8(0.2-28.2)$ & $7.2(0.5-26)$ \\
\hline Hemog & bin $(g / d L)$ & $9.9(5.5-15.3)$ & $9.6(5.5-14.6)$ & $9.9(6.3-14.6)$ & $9.7(5.8-14.8)$ \\
\hline TSH & & $3.9(<0.01-126)$ & $3.27(0.03-77)$ & $2(0.2-5.1)$ & $5.18(<0.01-126)^{\mathrm{a}}$ \\
\hline Neutrc & hils $/ \mu \mathrm{L}$ ascites & $3.6(0.25-31.4)$ & $3.9(0.26-31.4)$ & $4.3(0.26-16.4)$ & I.8I $(0.25-9.94)^{\mathrm{a}}$ \\
\hline Clinic & courses & & & & \\
\hline $\begin{array}{l}\text { Hepati } \\
\text { enceph }\end{array}$ & lopathy & $88(66.2 \%)$ & 50 (69.4\%) & 15 (68.2\%) & 35 (60.3\%) \\
\hline Varicea & bleeding & 49 (36.8\%) & 30 (4I.7\%) & 9 (40.9\%) & 18 (3।\%) \\
\hline $\begin{array}{l}\text { Hepatc } \\
\text { syndro }\end{array}$ & enal & 29 (21.8\%) & $16(22.2 \%)$ & $6(27.3 \%)$ & II (20\%) \\
\hline Portal & in thrombosis & 25 (18.8\%) & 17 (23.6\%) & 5 (22.7\%) & $9(15.5 \%)$ \\
\hline $\mathrm{HCC}$ & & $25(18.8 \%)$ & $7(9.7 \%)^{\mathrm{a}}$ & I (4.5\%) & $14(24.1 \%)$ \\
\hline Liver tr & nsplantation $^{d}$ & $9(6.8 \%)$ & $9(12.5 \%)^{\mathrm{a}}$ & $3(13.6 \%)$ & $\mathrm{I}(\mathrm{I} .7 \%)$ \\
\hline Sepsis & $\begin{array}{l}\text { At SBP } \\
\text { diagnosis }\end{array}$ & 9 (6.8\%) & 7 (9.7\%) & 5 (22.7\%) & I (I.7\%) \\
\hline & Overall & 29 (21.8\%) & $21(29.2 \%)^{a}$ & $12(54.5)^{\mathrm{a}}$ & $6(10.3 \%)$ \\
\hline ICU & $\begin{array}{l}\text { At SBP } \\
\text { diagnosis }\end{array}$ & 67 (50.4\%) & 39 (54.2\%) & $10(45.5 \%)$ & 27 (46.6\%) \\
\hline & Overall & 104 (78.2\%) & $62(86.1 \%)^{\mathrm{a}}$ & 20 (90.9\%) & 38 (65.5\%) \\
\hline Death & Overall & $58(43.6 \%)$ & 33 (45.8\%) & $15(68.2 \%)^{a}$ & 22 (38\%) \\
\hline & $\begin{array}{l}\text { Due to } \\
\text { sepsis }\end{array}$ & 18 (I3.5\%) & $12(16.7 \%)$ & $8(36.4 \%)^{a}$ & $3(5.2 \%)^{\mathrm{a}}$ \\
\hline & $\begin{array}{l}\text { Due to } \\
\text { others }\end{array}$ & 40 (30.1\%) & $2 \mathrm{I}(29.2 \%)$ & 7 (3I.2\%) & $19(32.8 \%)$ \\
\hline
\end{tabular}


Table I (Continued)

\begin{tabular}{llll}
\hline & All patients & $\begin{array}{l}\text { Patients with } \\
\text { MDROs }\end{array}$ & $\begin{array}{l}\text { Patients with } \\
\text { MDRO-SBP }\end{array}$ \\
\hline Death after 30 days & $33(24.8 \%)$ & $19(26.4 \%)$ & $9(40.9 \%)$ \\
Death after 90 days & $37(27.8 \%)$ & $22(30.6 \%)$ & $10(45.5 \%)$ \\
\hline
\end{tabular}

Notes: ${ }^{a}$ Significant difference $(P<0.05)$ was detected in these patients compared to patients without the detection of MDROs, without the detection of MDRO-SBP and with culture-positive SBP. ${ }^{\mathrm{b}} \mathrm{C}$ irrhosis was both alcoholic and due to viral hepatitis in eleven patients, resulting in I46 etiologies in total in this line. ${ }^{\mathrm{c} C i r r h o s i s}$ due to other reasons composed of primary sclerosing cholangitis, autoimmune hepatitis, alpha I-antitrypsin deficiency, and cirrhosis due to both HBV and cardiac cirrhosis, with $\mathrm{n}=\mathrm{I}$ each. ${ }^{\mathrm{d}} \mathrm{Two}$ patients suffered from newly occurring cirrhosis of the liver transplant eleven and 19 years after transplantation, respectively. ${ }^{e}$ Death due to other reasons composed of liver failure (ten patients), multi-organ failure (six patients), lactic acidosis (five patients), gastrointestinal bleeding (four patients), bleeding other than gastrointestinal (six patients), respiratory failure (four patients), kidney failure (two patients), liver transplant failure and heart failure (one patient each). MDRO-SBP, SBP with the evidence of MDROs or S. maltophilia in ascitic culture.

Abbreviations: ALBI, Albumin-Bilirubin; CLIF-AD, Chronic Liver Failure-Acute Decompensation; CRP, C-reactive protein; HCC, hepatocellular carcinoma; ICU, intensive care unit; MDRO, multidrug-resistant organism; MELD, Model for End-stage Liver Disease; SBP, spontaneous bacterial peritonitis; S. maltophilia, Stenotrophomonas maltophilia; $\mathrm{TSH}$, thyroid-stimulating hormone.

in 22 patients tested positive for MDRGN and VRE, one patient for MRSA and VRE and one patient with MDRGN, MRSA and VRE.

\section{Microbiological characterization of SBP and MDRO-SBP}

Ascitic culture was positive in 60/133 patients (45.1\%) at first paracentesis and in 75/133 patients (56.4\%) altogether. Grampositive bacteria were detected in $29 / 75$ patients $(38.7 \%)$, followed by gram-negative bacteria in $25 / 75$ patients $(33.3 \%)$. Both gram-positive and gram-negative bacteria were found in $21 / 75$ patients $(28 \%)$, which were due to sequential detection in $10 / 21$ patients $(47.6 \%)$ and simultaneous, ie, polymicrobial SBP, in $11 / 21$ patients $(52.4 \%)$. In these patients, abdominal perforation as a cause for peritonitis had been ruled out by computed tomography. Appropriate initial antibiotic therapy according to ascitic resistance profile was administered in $41 / 60$ patients $(68.3 \%)$ with positive first ascitic culture. Patients carrying MDROs yielded positive ascitic cultures more frequently $(52 / 72$ cases, $72.2 \%$; $\mathrm{HR}=14.6, P<0.001)$, demonstrating that in these patients ascitic MDRO presence was also more likely to be detected.

In 22/133 patients, MDROs or S. maltophilia were detected in ascitic cultures during at least one episode. This was the case upon the first diagnosis of SBP in 15/22 patients $(68.2 \%)$, while MDRO-SBP as a recurring episode of SBP occurred in $7 / 22$ patients $(31.8 \%)$ with an estimated time-dependent probability of 14\% (standard error $[\mathrm{SE}]=1.4 \%)$ after 60 days and $28 \%(\mathrm{SE}=2 \%)$ after 90 days, respectively. In the cases of MDRO-SBP after study inclusion, mean MELD score did not differ from baseline MELD. MDRO-SBP was nosocomial in 15/22 cases (68.2\%). In 13/22 patients with MDRO-SBP (59.1\%), further MDROs were detected in other compartments than ascites (Table S2).

\section{Assessing the occurrence of MDRO-SBP upon rectal screening}

Since SBP originates from the intestinal microflora, we reasoned that rectal $\mathrm{MDRO}$ screening might reveal causative to pathogens in patients with MDRO-SBP. Among all patients with MDRO-positive ascitic culture, the identical strain could be detected in other body compartments; rectal swabs being the most frequent localization with 17/22 cases (77.3\%; Table 3). Rectal, cutaneous or nasopharyngeal screening identified the corresponding pathogens in $20 / 22$ cases $(90.9 \%)$. Of note, one of these did not undergo rectal MDRO screening. The estimated time-dependent probability for the occurrence of MDRO-SBP upon the first positive rectal MDRO screening was $11.5 \%$ ( $\mathrm{SE}=2.8 \%$ ) after 30 days and $12.5 \%(\mathrm{SE}=3 \%)$ after 60 days, respectively. Time-dependent sensitivity of MDRO screening before the occurrence of MDRO-SBP was $77 \%$ after 30 days and $87 \%$ after 90 days, while negative predictive value (NPV) was $83 \%$ and $76 \%$, respectively (Table S3; Figure S2).

\section{Antibiotic treatment and secondary prophylaxis}

Standard empirical therapy for community-acquired SBP at UHF is intravenous ceftriaxone, while the standard of care for nosocomial SBP is a carbapenem such as meropenem or imipenem. Based on the individual microbial data, diverging antibiotic regimens may have been chosen, and inappropriate initial therapy resulted in decreased survival in 19/60 (31.7\%) patients with culture-positive SBP (HR $=1.92, P=0.014)$. Initial treatment of MDRO-SBP comprised of carbapenems 

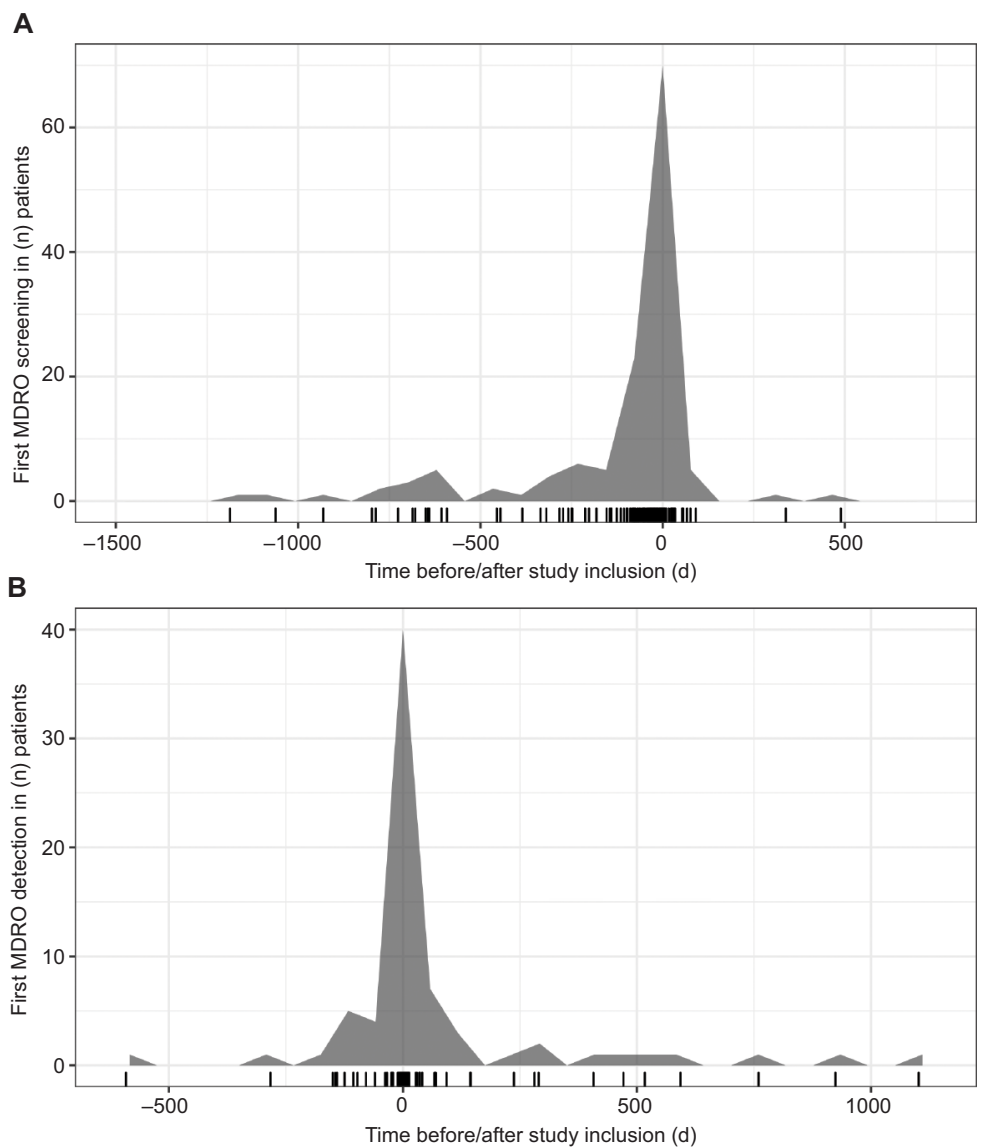

Figure 2 (A) Time of first in-house MDRO screening in relation to time of study inclusion. Ticks at the bottom represent first rectal swab. Screening was performed before or within 28 days upon study inclusion in 123 patients (92.5\%), and later than 28 days upon inclusion in 10 patients (7.5\%). (B) Time of first MDRO detection in relation to time of study inclusion. First overall diagnosis of MDRO had been made before study inclusion in 24/72 patients $(33.3 \%)$ and was made upon or after study inclusion in $48 / 72$ patients $(66.7 \%)$.

Abbreviations: E. coli, Escherichia coli; ESBL, extended-spectrum B-lactamase; ICU, intensive care unit; IMU, intermediate care unit; MDRO, multidrug-resistant organism; QR, fluoroquinolone resistance; SBP, spontaneous bacterial peritonitis; S. maltophilia, Stenotrophomonas maltophilia.

Table 2 Cumulative evidence of MDROs and S. maltophilia collected during the observational period, arranged by sample type and strain

\begin{tabular}{|c|c|c|c|c|c|c|c|c|}
\hline \multirow{2}{*}{\multicolumn{2}{|c|}{$\begin{array}{l}\text { MDROs; } \sum=72 \text { patients }{ }^{\mathrm{a}} \\
\text { MDRGN (42 patients, } 3 \text { I.6\%) }\end{array}$}} & \multicolumn{7}{|c|}{ Material } \\
\hline & & \multirow{2}{*}{$\frac{\text { Ascites }}{8(11 \%)}$} & \multirow{2}{*}{$\frac{\text { Blood }}{3(4 \%)}$} & \multirow{2}{*}{$\frac{\text { Rectal }}{16(22 \%)}$} & \multirow{2}{*}{$\begin{array}{l}\text { Throat/skin/nose } \\
2(3 \%)\end{array}$} & \multirow{2}{*}{$\frac{\text { Urine }}{2(3 \%)}$} & \multirow{2}{*}{ Wound } & \multirow{2}{*}{$\begin{array}{l}\text { Others } \\
\mathrm{I}(\mathrm{I} \%)\end{array}$} \\
\hline E. coli & $\mathrm{ESBL}+\mathrm{QR}$ & & & & & & & \\
\hline & ESBL & $2(3 \%)$ & - & $3(4 \%)$ & - & I (I\%) & - & - \\
\hline \multirow[t]{3}{*}{ K. pneumoniae } & $\mathrm{ESBL}+\mathrm{QR}$ & - & $2(3 \%)$ & $7(10 \%)$ & $2(3 \%)$ & $\mathrm{I}(1 \%)$ & - & - \\
\hline & $C R+Q R$ & - & - & $2(3 \%)$ & - & - & - & - \\
\hline & ESBL & $2(3 \%)$ & $2(3 \%)$ & $4(6 \%)$ & $4(6 \%)$ & - & I (I\%) & I (I\%) \\
\hline P. aeruginosa & $C R+Q R$ & - & - & I (I\%) & I (I\%) & I (I\%) & - & - \\
\hline \multirow[t]{2}{*}{ E. cloacae } & CephR & - & - & - & - & I (I\%) & - & - \\
\hline & CephR/QR & - & - & $2(3 \%)$ & - & $2(3 \%)$ & - & - \\
\hline \multirow[t]{2}{*}{ C. freundii } & CephR & - & - & $2(3 \%)$ & - & - & - & - \\
\hline & CephR + QR & - & - & I (I\%) & - & - & - & - \\
\hline S. maltophilia & No MDROs & $2(3 \%)$ & - & I (I\%) & - & - & - & - \\
\hline \multicolumn{2}{|c|}{ VRE (50 patients, $37.6 \%)$} & $8(11 \%)$ & $\mathrm{I}(\mathrm{I} \%)$ & $47(65 \%)$ & $2(3 \%)$ & $3(4 \%)$ & $2(3 \%)$ & I (I\%) \\
\hline \multicolumn{2}{|c|}{ MRSA (5 patients, $3.8 \%$ ) } & I (I\%) & - & $\mathrm{I}(\mathrm{I} \%)$ & $7(10 \%)$ & $\mathrm{I}(\mathrm{I} \%)$ & $2(3 \%)$ & - \\
\hline
\end{tabular}

Notes: One patient was positive for both ESBL + QR E. coli and VRE in ascitic cultures, resulting in the detection of 23 ascitic MDROs in total. Of note, S. maltophilia was not genuinely classified as MDRO, but was nevertheless considered a relevant pathogen due to its intrinsic resistance to CRs. Enterobacteriaceae with ESBL phenotype as well as Enterobacteriaceae, $P$. aeruginosa and A. baumannii resistant to piperacillin, any third-/fourth-generation cephalosporins and QR \pm CR. aMultiple MDRO strains were detected in 24 individuals, resulting in 22 patients testing positive for MDRGN and VRE, one patient for MRSA and VRE and one patient with MDRGN, MRSA and VRE.

Abbreviations: A. baumannii, Acinetobacter baumannii; CephR, cephalosporine resistance; $C$. freundii, Citrobacter freundii; CR, carbapenem resistance; $E$. cloacae, Enterobacter cloacae; E. coli, Escherichia coli; ESBL, extended-spectrum B-lactamase; K. pneumoniae, Klebsiella pneumoniae; MDRGN, multidrug-resistant gram-negative bacteria; MDRO, multidrug-resistant organism; MRSA, methicillin-resistant Streptococcus aureus; P. aeruginosa, Pseudomonas aeruginosa; QR, fluoroquinolone resistance; S. maltophilia, Stenotrophomonas maltophilia; VRE, vancomycin-resistant enterococci. 
Table 3 Colocalizations of MDROs and S. maltophilia in other body compartments in patients with MDRO-SBP

\begin{tabular}{|c|c|c|c|c|c|c|}
\hline \multirow[t]{2}{*}{ MDRO-SBP $(n=2 I)$} & \multicolumn{6}{|c|}{ Material } \\
\hline & $\begin{array}{l}\text { Rectal } \\
(n=18)\end{array}$ & $\begin{array}{l}\text { Throat/ } \\
\text { skin }(n=2)\end{array}$ & $\begin{array}{l}\text { Blood } \\
(n=5)\end{array}$ & $\begin{array}{l}\text { Body fluida } \\
(n=3)\end{array}$ & $\begin{array}{l}\text { Wound/ } \\
\text { surgical }(n=3)\end{array}$ & $\begin{array}{l}\text { Deviceb }^{b} \\
(n=2)\end{array}$ \\
\hline \multicolumn{7}{|c|}{ Gram-positive pathogens $(n=8)$} \\
\hline $\operatorname{VRE}(n=7)$ & 6 & - & I & - & 1 & - \\
\hline MRSA $(n=1)$ & I & 1 & - & - & 1 & 1 \\
\hline \multicolumn{7}{|c|}{$\begin{array}{l}\text { MDRGN and S. maltophilia } \\
(n=13)\end{array}$} \\
\hline K. pneumoniae ESBL $(n=2)$ & I & - & 2 & - & - & 1 \\
\hline E. coli $(n=9 c)$ & 7 & 1 & 2 & 1 & 1 & - \\
\hline S. maltophilia $(\mathrm{n}=2)$ & I & - & - & 1 & - & - \\
\hline \multicolumn{7}{|l|}{ VRE + MDRGN $(n=1)$} \\
\hline E. coli $\mathrm{ESBL}+\mathrm{QR}$ & I & - & - & - & - & - \\
\hline VRE & I & - & - & 1 & - & - \\
\hline
\end{tabular}

Notes: Of note, strains were detected only in ascitic cultures, and the abovementioned sites are given. One patient was excluded from the chart due to incomplete MDRO

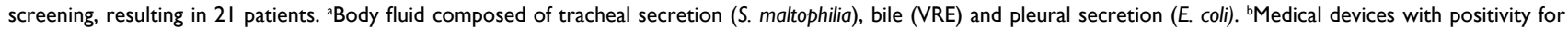
MDROs composed of left ventricular assistant device (MRSA) and Shaldon catheter ( $K$. pneumoniae). 'Patients with MDRO E. coli composed of $n=2$ expressing ESBL and $n=7$ expressing ESBL + QR. MDRO-SBP, SBP with the evidence of MDROs or S. maltophilia in ascitic culture.

Abbreviations: E. coli, Escherichia coli; ESBL, extended-spectrum B-lactamase; K. pneumoniae, Klebsiella pneumoniae; MDRGN, multidrug-resistant gram-negative bacteria; MDRO, multidrug-resistant organism; MRSA, methicillin-resistant Streptococcus aureus; QR, fluoroquinolone resistance; SBP, spontaneous bacterial peritonitis; S. maltophilia, Stenotrophomonas maltophilia; VRE, vancomycin-resistant enterococci.

in $16 / 22$ patients $(72.3 \%)$, cephalosporins and piperacillin/ tazobactam in $2 / 22$ patients $(9.1 \%$ each), and a fluoroquinolone in one patient $(4.5 \%)$. Initial treatment furthermore composed of glycopeptides, oxazolidinones or tigecycline in four of eight patients with the diagnosis of VRE-associated MDRO-SBP. Among these cases, resistance against one or more of these agents was detected in four of eight patients (50\%; Table 4), while all cases of MDRO-SBP associated with VRE displayed QR expression. If necessary, antibiotic regimens were adapted immediately according to microbiological findings. Nevertheless, inadequate initial antibiotic therapy was associated with highly increased risk of death in MDRO-SBP patients (14/22 patients, $63.6 \%$, HR $=11.4$, $P=0.002$ ). Clinical courses did not differ notably between different causatives of MDRO-SBP (Table S4).

One hundred and five patients (78.9\%) could be discharged after first inpatient treatment. Secondary antibiotic prophylaxis as recommended by the current hepatologic guidelines ${ }^{34}$ was prescribed in $86 / 105$ patients $(81.9 \%$; Table S5). Recurrent SBP occurred in 35/105 patients (33.3\%), and ascitic evidence of bacteria expressing QR was found in 13 of these patients $(37.1 \%)$ and in 36/133 patients in total $(27.1 \%)$. This was due to Enterococcus spp. or VRE in $16 / 36$ cases $(44.4 \%)$, MDRGN in $8 / 36$ cases $(22.2 \%)$, MRSA in one case (2.8\%) and due to others (other pathogens expressing QR composed of coagulase-negative Staphylococcus spp. [four patients], K. pneumoniae [two patients] and Clostridium tertium, E. coli, S. aureus, Streptococcus mitis and S. maltophilia [one patient each]) in 11/36 cases (30.6\%). The estimated time-dependent probabilities for recurring $\mathrm{SBP}$ were $9.6 \%(\mathrm{SE}=2.8 \%)$ after 30 days, $15.7 \%$ $(\mathrm{SE}=3.5 \%)$ after 60 days and $17.9 \%(\mathrm{SE}=3.8 \%)$ after 90 days, and MDRO-SBP was more likely to occur in patients with recurrent $\mathrm{SBP}(\mathrm{HR}=2.82, P=0.001)$.

\section{Discussion}

The global incidence of MDRO-SBP, especially caused by pathogens expressing resistance to cephalosporins and fluoroquinolones, is a growing health threat. ${ }^{36}$ We showed that accurate microbiological screening measures helped identify patients who developed MDRO-SBP. These patients are at high risk of death due to sepsis. Although the study was designed in a retrospective fashion, our findings are consistent and clinically substantial due to the following arguments.

First, patients with and without MDRO detection had similar baseline parameters, thus resembling a homogenous population in terms of the severity of disease. Second, survival was impaired if the initial empiric antibiotic therapy did not cover the indicated pathogens, which is in line with previous findings and shows that lethality was mainly associated with bacterial infections. ${ }^{37,38}$ Third, while liver scores and survival in patients with culture-negative SBP were comparable to culture-positive patients, lethal sepsis was less frequent (Table 1).

Regarding patients with culture-negative SBP, one might argue that cases of ascitic MDRO presence might 
Table 4 Distribution of resistance patterns among causatives of MDRO-SBP

\begin{tabular}{|c|c|c|c|c|c|}
\hline \multirow[t]{2}{*}{ Pathogen } & \multicolumn{5}{|c|}{ Resistance pattern } \\
\hline & ESBL & CR & QR & Amikacin/colistin & Teicoplanin $\left( \pm\right.$ tigecycline/linezolid $\left.{ }^{a}\right)$ \\
\hline E. coli $(\mathrm{n}=10)$ & 10 & 0 & 8 & 0 & - \\
\hline K. pneumoniae $(n=2)$ & 2 & 0 & 0 & 0 & - \\
\hline $\operatorname{VRE}(n=8)$ & - & - & 8 & - & 4 \\
\hline
\end{tabular}

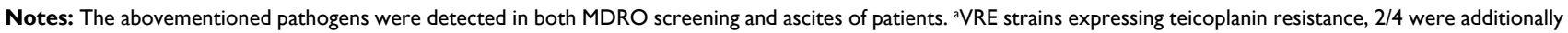
resistant to tigecycline and 2/4 to linezolid. Of note, cases of S. maltophilia are not included in this table. MDRO-SBP, SBP with the evidence of MDROs or S. maltophilia in ascitic culture.

Abbreviations: CR, carbapenem resistance; E. coli, Escherichia coli; ESBL, extended-spectrum B-lactamase; K. pneumoniae, Klebsiella pneumoniae; MDRO, multidrug-resistant organism; QR, fluoroquinolone resistance; SBP, spontaneous bacterial peritonitis; S. maltophilia, Stenotrophomonas maltophilia; VRE, vancomycin-resistant enterococci.

have been missed in some of these patients. However, It is well known that the sensitivity of ascitic cultures is only around $50 \%,{ }^{18}$ a fact that is reflected by our findings and has to be dealt with throughout the management of SBP. Nevertheless, MDRO-SBP was exclusively detected in patients with carriage of MDROs in other body compartments as well, while patients negative for MDROs also had significantly less positive ascitic cultures $(\mathrm{HR}=0.07, P<0.001)$. Considering the significantly better outcome in regard of septic complications in these patients, who are regularly treated with ceftriaxone, these SBP cases have to be considered as nonresistant rather than resistant.

Time-dependent sensitivity of MDRO screening for developing MDRO-SBP was between $77 \%$ and $95 \%$ including a high NPV, while specificity of the test was only around $50 \%$ (Table S3). Thus, negative MDRO screening might rule out MDRO-SBP with a high likelihood. Due to its low specificity and positive predictive value (PPV), though, repetitive diagnostic paracentesis including microbiological sample collection remains mandatory. Patients with nosocomial SBP should receive empiric antibiotic therapy according to local resistance patterns. ${ }^{28}$ A more "narrow" antibiotic therapy might be justified if MDRO screening was negative, but prospective data are warranted. Notably, the first MDRO evidence was made by the time of diagnosis of MDRO-SBP in almost half of the patients, since no screening results had been available beforehand.

Based on these observations, the ascitic detection of MDROs resembles a major prognostic factor in patients with decompensated cirrhosis. ${ }^{11,36}$ Because these patients are afflicted by rapid deterioration and death, early MDRO detection is vital. ${ }^{37,38}$ Since directional spread of MDROs goes from the intestine or skin into the abdomen (and not vice versa), it would be appropriate to screen for MDRO colonization in patients with risk or even history of SBP. Our data give the first evidence that this approach might be valid in the clinical setting and suggest that MDRO screening should be conducted on the day of patients' admission. However, a study including a larger sample size of patients with MDRO-SBP is warranted.

\section{Conclusion}

Due to the retrospective design of the study, it must be stressed that prospective data are warranted to validate the accuracy of MDRO screening in association with MDROSBP. Therefore, general treatment recommendations cannot be made based on our findings. Furthermore, patients were mainly recruited from ICU/IMC. This translates into comparably high liver scores upon inclusion and an overall reduced survival during inpatient treatment as well as under secondary prophylaxis. ${ }^{39}$ Yet, differences between patients with and without MDRO-SBP were significant and comprehensible. Importantly, microbiological resistance data might depend on local resistance patterns, which is reflected by a high proportion of VRE, and may therefore not be transferable unrestrictedly. However, our microbiological data are comparable to reports from other monocenter studies..$^{1,2,11-14,21,22,40}$ On a more general basis, we suggest a prospective multicenter setting to determine the long-term clinical impact of MDRO-SBP and the benefit of MDRO screening in patients with decompensated cirrhosis.

\section{Author contributions}

Compiling data, data interpretation and drafting and writing the manuscript: PGF, MM and OW: Biostatistical analysis and interpretation: PGF and NF. Data interpretation and critical review of the manuscript: SZ, VAJK, MH, TAW, CML, JV, $\mathrm{CR}$ and $\mathrm{OW}$. All the authors approved the final version of the manuscript and agreed to be accountable for all aspects of 
the work in ensuring that questions related to the accuracy or integrity of any part of the work are appropriately investigated and resolved. All authors contributed toward data analysis, drafting and critically revising the paper and agree to be accountable for all aspects of the work.

\section{Disclosure}

SZ has provided consultancy for Abbvie, BMS, Gilead, Intercept, Janssen and Merck (all outside the scope of this study). OW has provided consultancy for Amgen, Bayer, BMS, Celgene, Eisai, Merck, Novartis, Roche, Servier and Shire (all outside the scope of this study). VAJK was partially supported by a grant from the Deutsche Forschungsgemeinschaft (DFG, research unit 2251). The authors report no other conflicts of interest in this work.

\section{References}

1. Tandon P, Delisle A, Topal JE, Garcia-Tsao G. High prevalence of antibiotic-resistant bacterial infections among patients with cirrhosis at a US liver center. Clin Gastroenterol Hepatol. 2012;10(11):1291-1298.

2. Fernández J, Acevedo J, Castro M, et al. Prevalence and risk factors of infections by multiresistant bacteria in cirrhosis: a prospective study. Hepatology. 2012;55(5):1551-1561.

3. Bajaj JS, O’Leary JG, Reddy KR, et al. Second infections independently increase mortality in hospitalized patients with cirrhosis: the North American consortium for the study of end-stage liver disease (NACSELD) experience. Hepatology. 2012;56(6):2328-2335.

4. Garcia-Tsao G. Current Management of the Complications of Cirrhosis and Portal Hypertension: Variceal Hemorrhage, Ascites, and Spontaneous Bacterial Peritonitis. Dig Dis. 2016;34(4):382-386.

5. Arvaniti V, D'Amico G, Fede G, et al. Infections in patients with cirrhosis increase mortality four-fold and should be used in determining prognosis. Gastroenterology. 2010;139(4):1246-1256.

6. Singal AK, Salameh H, Kamath PS. Prevalence and in-hospital mortality trends of infections among patients with cirrhosis: a nationwide study of hospitalised patients in the United States. Aliment Pharmacol Ther. 2014;40(1):105-112.

7. Ferstl PG, Filmann N, Brandt C, et al. The impact of carbapenem resistance on clinical deterioration and mortality in patients with liver disease. Liver Int. 2017;37(10):1488-1496.

8. Waidmann O, KempfVA, Brandt C, Zeuzem S, Piiper A, Kronenberger B. Colonisation with multidrug-resistant bacteria is associated with increased mortality in patients with cirrhosis. Gut. 2015;64(7):1183-1184.

9. Salerno F, Borzio M, Pedicino C, et al. The impact of infection by multidrug-resistant agents in patients with cirrhosis. A multicenter prospective study. Liver Int. 2017;37(1):71-79.

10. Lutz P, Nischalke HD, Strassburg CP, Spengler U. Spontaneous bacterial peritonitis: The clinical challenge of a leaky gut and a cirrhotic liver. World J Hepatol. 2015;7(3):304-314.

11. Lutz P, Nischalke HD, Krämer B, et al. Antibiotic resistance in healthcare-related and nosocomial spontaneous bacterial peritonitis. Eur $J$ Clin Invest. 2017;47(1):44-52.

12. Merli M, Lucidi C, di Gregorio V, et al. The spread of multi drug resistant infections is leading to an increase in the empirical antibiotic treatment failure in cirrhosis: a prospective survey. PLoS One. 2015;10(5):e0127448.

13. Alexopoulou A, Vasilieva L, Agiasotelli D, et al. Extensively drugresistant bacteria are an independent predictive factor of mortality in 130 patients with spontaneous bacterial peritonitis or spontaneous bacteremia. World J Gastroenterol. 2016;22(15):4049-4056.
14. Ariza X, Castellote J, Lora-Tamayo J, et al. Risk factors for resistance to ceftriaxone and its impact on mortality in community, healthcare and nosocomial spontaneous bacterial peritonitis. $J$ Hepatol. 2012;56(4):825-832.

15. Fernández J, Navasa M, Gómez J, et al. Bacterial infections in cirrhosis: epidemiological changes with invasive procedures and norfloxacin prophylaxis. Hepatology. 2002;35(1):140-148.

16. Ginés P, Rimola A, Planas R, et al. Norfloxacin prevents spontaneous bacterial peritonitis recurrence in cirrhosis: results of a double-blind, placebo-controlled trial. Hepatology. 1990;12(4 Pt 1):716-724.

17. Fernández J, Tandon P, Mensa J, Garcia-Tsao G. Antibiotic prophylaxis in cirrhosis: Good and bad. Hepatology. 2016;63(6):2019-2031.

18. Jalan R, Fernandez J, Wiest R, et al. Bacterial infections in cirrhosis: a position statement based on the EASL Special Conference 2013. $J$ Hepatol. 2014;60(6):1310-1324.

19. Cobos-Trigueros N, Solé M, Castro P, et al. Acquisition of Pseudomonas aeruginosa and its resistance phenotypes in critically ill medical patients: role of colonization pressure and antibiotic exposure. Crit Care. 2015;19:218.

20. Winter SE, Bäumler AJ. Dysbiosis in the inflamed intestine: chance favors the prepared microbe. Gut Microbes. 2014;5(1):71-73.

21. Reuken PA, Pletz MW, Baier M, Pfister W, Stallmach A, Bruns T. Emergence of spontaneous bacterial peritonitis due to enterococci - risk factors and outcome in a 12-year retrospective study. Aliment Pharmacol Ther. 2012;35(10):1199-1208.

22. Fernández J, Bert F, Nicolas-Chanoine MH. The challenges of multidrug-resistance in hepatology. J Hepatol. 2016;65(5):1043-1054.

23. Pan SC, Wang JT, Chen YC, Chang YY, Chen ML, Chang SC. Incidence of and risk factors for infection or colonization of vancomycinresistant enterococci in patients in the intensive care unit. PLoS One. 2012;7(10):e47297.

24. Fernández J, Acevedo J. New antibiotic strategies in patients with cirrhosis and bacterial infection. Expert Rev Gastroenterol Hepatol. 2015;9(12):1495-1500.

25. Acevedo J, Silva A, Prado V, Fernández J. The new epidemiology of nosocomial bacterial infections in cirrhosis: therapeutical implications. Hepatol Int. 2013;7(1):72-79.

26. European Association for the Study of the Liver. EASL clinical practice guidelines on the management of ascites, spontaneous bacterial peritonitis, and hepatorenal syndrome in cirrhosis. $J$ Hepatol. 2010;53(3):397-417.

27. Cheong HS, Kang CI, Lee JA, et al. Clinical significance and outcome of nosocomial acquisition of spontaneous bacterial peritonitis in patients with liver cirrhosis. Clin Infect Dis. 2009;48(9):1230-1236.

28. Piano S, Fasolato S, Salinas F, et al. The empirical antibiotic treatment of nosocomial spontaneous bacterial peritonitis: Results of a randomized, controlled clinical trial. Hepatology. 2016;63(4): 1299-1309.

29. Bundesministerium der Justiz und für Verbraucherschutz Bundesministerium der Justiz und für Verbraucherschutz, 2000 [homepage on the Internet].Gesetz zur Verhütung und Bekämpfung von Infektionskrankheiten beim Menschen (Infektionsschutzgesetz - IfSG). Federal Ministry Justice and Consumer Protection, 2000. Law on Prevention and Control of Infectious Diseases in Humans (Infection Protection Act - IfSG). Available from: http://www.gesetze-im-internet.de/ifsg/ BJNR104510000.html. Accessed September 5, 2018. German.

30. Bundesministerium der Justiz und für Verbraucherschutz Bundesministerium der Justiz und für Verbraucherschutz [homepage on the Internet]. Gesetz zur Verhütung und Bekämpfung von Infektionskrankheiten beim Menschen (Infektionsschutzgesetz - IfSG). Law on Prevention and Control of Infectious Diseases in Humans (Infection Protection Act - IfSG). Federal Ministry Justice and Consumer Protection; 2000]. Available from: http://www.gesetze-im-internet.de/ifsg/BJNR104510000.html. Accessed September 5, 2018. German.

31. Reinheimer C, Kempf VA, Göttig S, et al. Multidrug-resistant organisms detected in refugee patients admitted to a University Hospital, Germany June-December 2015. Euro Surveill. 2016;21(2):30110. 
32. Magiorakos AP, Srinivasan A, Carey RB, et al. Multidrug-resistant, extensively drug-resistant and pandrug-resistant bacteria: an international expert proposal for interim standard definitions for acquired resistance. Clin Microbiol Infect. 2012;18(3):268-281.

33. Doyle D, Peirano G, Lascols C, Lloyd T, Church DL, Pitout JD. Laboratory detection of Enterobacteriaceae that produce carbapenemases. $J$ Clin Microbiol. 2012;50(12):3877-3880.

34. Higgins PG, Dammhayn C, Hackel M, Seifert H. Global spread of carbapenem-resistant Acinetobacter baumannii. J Antimicrob Chemother. 2010;65(2):233-238.

35. Blanche P, Dartigues JF, Jacqmin-Gadda H. Estimating and comparing time-dependent areas under receiver operating characteristic curves for censored event times with competing risks. Stat Med. 2013;32(30):5381-5397.

36. Fiore M, Maraolo AE, Gentile I, et al. Nosocomial spontaneous bacterial peritonitis antibiotic treatment in the era of multi-drug resistance pathogens: A systematic review. World $J$ Gastroenterol. 2017;23(25):4654-4660
37. Umgelter A, Reindl W, Miedaner M, Schmid RM, Huber W. Failure of current antibiotic first-line regimens and mortality in hospitalized patients with spontaneous bacterial peritonitis. Infection. 2009;37(1):2-8.

38. Friedrich-Rust M, Wanger B, Heupel F, et al. Influence of antibioticregimens on intensive-care unit-mortality and liver-cirrhosis as risk factor. World J Gastroenterol. 2016;22(16):4201-4210.

39. Elfert A, Abo Ali L, Soliman S, Ibrahim S, Abd-Elsalam S. Randomizedcontrolled trial of rifaximin versus norfloxacin for secondary prophylaxis of spontaneous bacterial peritonitis. Eur J Gastroenterol Hepatol. 2016;28(12):1450-1454.

40. Reuken PA, Pletz MW, Baier M, Pfister W, Stallmach A, Bruns T. Emergence of spontaneous bacterial peritonitis due to enterococci - risk factors and outcome in a 12-year retrospective study. Aliment Pharmacol Ther. 2012;35(10):1199-1208. 


\section{Supplementary materials}

Impact of multidrug-resistant organisms

in spontaneous bacterial peritonitis

Table SI Liver scores at study inclusion, arranged by clinical end points

\begin{tabular}{|c|c|c|c|c|c|c|c|}
\hline \multirow[t]{2}{*}{ Score } & \multicolumn{3}{|c|}{ Death $(n=58)$} & \multicolumn{4}{|c|}{ Lost to follow up $(n=75)$} \\
\hline & Mean & Median & SD & Mean & Median & SD & $P$-value \\
\hline MELD & 25.6 & 25 & 8.57 & 19.9 & 19 & 7.23 & 0.0001 \\
\hline CLIF-AD & 64.8 & 67 & 10.20 & 59.7 & 58 & 10.66 & 0.007 \\
\hline ALBI & 1.249 & 1.23 & 0.395 & 1.070 & 1.050 & 0.301 & 0.005 \\
\hline
\end{tabular}

Abbreviations: ALBI, Albumin-Bilirubin; CLIF-AD, Chronic Liver Failure-Acute Decompensation; MELD, Model for End-stage Liver Disease.

Table S2 Timeline and additional microbiological findings in patients with MDRO-SBP

\begin{tabular}{|c|c|c|c|c|c|}
\hline Patient & MDRO-SBP & Further MDROs & $\begin{array}{l}\text { Further ascitic } \\
\text { detections }\end{array}$ & $\begin{array}{l}\text { Time in } \\
\text { study } \\
\text { (days) }\end{array}$ & $\begin{array}{l}\text { Time to } \\
\text { MDRO- } \\
\text { SBP (days) }\end{array}$ \\
\hline I & E. coli $\mathrm{ESBL}+\mathrm{QR}$ & - & S. aureus & 23 & 0 \\
\hline 2 & $\begin{array}{l}\text { K. pneumoniae } \\
\text { ESBL }\end{array}$ & - & - & 116 & 111 \\
\hline 3 & $\begin{array}{l}\text { E. coli ESBL + } \\
\text { QR, VRE }\end{array}$ & K. pneumoniae ESBL+QR (rectal) & E. cloacae CephR/QR & 697 & 593 \\
\hline 4 & VRE & - & $\begin{array}{l}\text { C. koseri, A. baumannii } \\
\text { P. putida, Candida famata }\end{array}$ & $|8|$ & 53 \\
\hline 5 & S. maltophilia & - & E. faecium, Candida albicans & 6 & 5 \\
\hline 6 & VRE & - & - & 7 & 0 \\
\hline 7 & VRE & - & $\begin{array}{l}\text { S. haemolyticus, Candida } \\
\text { albicans }\end{array}$ & 7 & 0 \\
\hline 8 & E. coli $\mathrm{ESBL}+\mathrm{QR}$ & VRE (rectal) & - & I & 0 \\
\hline 9 & E. coli ESBL & - & - & 4 & 0 \\
\hline 10 & MRSA & $\begin{array}{l}\text { E. coli (rectal) } \\
\text { VRE (rectal, wound) } \\
\text { K. pneumoniae (rectal, medical device) }\end{array}$ & - & 390 & 0 \\
\hline II & E. coli $\mathrm{ESBL}+\mathrm{QR}$ & $\begin{array}{l}\text { VRE (rectal) } \\
\text { E. cloacae (urine) }\end{array}$ & $\begin{array}{l}\text { Ent. hirae, } B . \\
\text { amyloliquefaciens }\end{array}$ & 799 & 94 \\
\hline 12 & VRE & $\begin{array}{l}\text { K. pneumoniae (rectal, throat, blood) } \\
\text { P. aeruginosa (tracheal secretion) }\end{array}$ & S. mitis, E. coli & 671 & 297 \\
\hline 13 & E. coli ESBL & - & - & 7 & 2 \\
\hline 14 & VRE & - & $\begin{array}{l}\text { P. rettgeri, S. aureus, B. } \\
\text { cereus, S. epidermidis, S. } \\
\text { haemolyticus }\end{array}$ & 14 & 8 \\
\hline 15 & VRE & - & E. coli & 14 & 10 \\
\hline 16 & $\begin{array}{l}\text { K. pneumoniae } \\
\text { ESBL }\end{array}$ & - & S. pneumoniae & 30 & 14 \\
\hline 17 & E. coli ESBL + QR & VRE & - & 72 & 67 \\
\hline 18 & E. coli $\mathrm{ESBL}+\mathrm{QR}$ & - & - & 15 & 0 \\
\hline 19 & S. maltophilia & VRE (rectal) & $\begin{array}{l}\text { S. parasanguinis, E. faecalis, } \\
\text { S. epidermidis }\end{array}$ & 352 & 303 \\
\hline 20 & E. coli $\mathrm{ESBL}+\mathrm{QR}$ & - & E. faecium & 96 & 0 \\
\hline 21 & VRE & E. coli ESBL+QR (rectal) & $\begin{array}{l}\text { E. gallinarum, E. faecium, } S \text {. } \\
\text { epidermidis }\end{array}$ & 73 & 8 \\
\hline 22 & E. coli ESBL + QR & VRE (rectal) & - & 265 & 0 \\
\hline
\end{tabular}

Note: MDRO-SBP, SBP with the evidence of MDROs or S. maltophilia in ascitic culture.

Abbreviations: CephR, cephalosporine resistance; E. cloacae, Enterobacter cloacae; E. coli, Escherichia coli; ESBL, extended-spectrum B-lactamase; K. pneumoniae, Klebsiella pneumoniae; MDRO, multidrug-resistant organism; MRSA, methicillin-resistant Streptococcus aureus; P. aeruginosa, Pseudomonas aeruginosa; QR, fluoroquinolone resistance; S. aureus, Streptococcus aureus; SBP, spontaneous bacterial peritonitis; S. maltophilia, Stenotrophomonas maltophilia; S. mitis, Streptococcus mitis; VRE, vancomycin-resistant enterococci; C. koseri, Citrobacter koseri; A. baumannii, Acinetobacter baumanni; P. putida, Pseudomonas putida; E. faecium, S. haemolyticus, Ent. Hirae, B. amyloliquefaciens, P. rettgeri, B. cereus, S. epidermidis, S. parasanguinis, E. faecalis, E. gallinarum. 
Table S3 Time-dependent sensitivity and specificity of rectal MDRO screening for the prediction of developing MDRO-SBP

\begin{tabular}{lllll}
\hline Time after first positive screening & Sensitivity (\%) & Specificity (\%) & PPV (\%) & NPV (\%) \\
\hline 30 days & 77 & 50 & 10 & 83 \\
90 days & 87 & 50 & 19 & 76 \\
I year & 92 & 62 & 34 & 62 \\
2 years & 95 & 71 & 52 & 48 \\
\hline
\end{tabular}

Notes: The values resemble the sensitivity and specificity of the test after the time given in the first column (time after the first positive screening). MDRO-SBP, SBP with evidence of MDROs or S. maltophilia in ascitic culture.

Abbreviations: MDRO, multidrug-resistant organism; NPV, negative predictive value; PPV, positive predictive value; SBP, spontaneous bacterial peritonitis; S. maltophilia, Stenotrophomonas maltophilia.

Table S4 Clinical courses of patients with MDRO-SBP, arranged by causative pathogens

\begin{tabular}{|c|c|c|c|c|c|c|}
\hline & & \multicolumn{5}{|c|}{22 patients with MDRO-SBP } \\
\hline & & $\begin{array}{l}\text { E. coli ESBL } \\
\pm Q R, n=10\end{array}$ & $\begin{array}{l}\text { K. pneumoniae } \\
\text { ESBL, } n=2\end{array}$ & $\begin{array}{l}\text { S. maltophilia, } \\
\mathrm{n}=2\end{array}$ & $\begin{array}{l}\text { VRE, } \\
n=8\end{array}$ & $\begin{array}{l}\text { MRSA, } \\
\mathrm{n}=\mathrm{I}\end{array}$ \\
\hline ICU & 9 & 6 & 2 & 2 & 8 & 1 \\
\hline Sepsis at SBP diagnosis & 2 & 1 & I & 1 & 2 & 0 \\
\hline Sepsis overall & 7 & 6 & 2 & 2 & 2 & 1 \\
\hline Death & 7 & 5 & 2 & 2 & 6 & I \\
\hline Death due to sepsis & 3 & 2 & 1 & 2 & 2 & I \\
\hline Death due to other reasons $s^{a}$ & 4 & 3 & I & 0 & 4 & 0 \\
\hline
\end{tabular}

Notes: Since in one patient MDRO-SBP due to both E. coli and VRE was detected, cumulative number of causative pathogens is 23. aDeath due to other reasons composed of lactic acidosis $(n=3)$, hemorrhagic shock due to variceal bleeding $(n=2)$, end-stage kidney failure $(n=l)$ and multi-organ failure $(n=l)$. MDRO-SBP, SBP with evidence of MDROs or S. maltophilia in ascitic culture.

Abbreviations: E. coli, Escherichia coli; ESBL, extended-spectrum B-lactamase; ICU, intensive care unit; K. pneumoniae, Klebsiella pneumoniae; MDRO, multidrug-resistant organism; MRSA, methicillin-resistant Streptococcus aureus; QR, fluoroquinolone resistance; SBP, spontaneous bacterial peritonitis; S. maltophilia, Stenotrophomonas maltophilia; VRE, vancomycin-resistant enterococci.

Table S5 Antibiotic prophylaxis or curative treatment in patients after SBP

\begin{tabular}{lll}
\hline & Death $(\mathbf{n}=\mathbf{3 0})$ & Lost to follow up $(\mathbf{n}=\mathbf{7 5})$ \\
\hline Quinolones $(\mathrm{n}=65)$ & $23(35.4 \%)$ & $42(64.6 \%)$ \\
Rifaximin $(\mathrm{n}=13)$ & $2(15.4 \%)$ & $1 \mathrm{I}(84.6 \%)$ \\
TIPS $(\mathrm{n}=9)$ & $2(22.2 \%)$ & $7(77.8 \%)$ \\
Liver transplantation $^{\mathrm{a}}(\mathrm{n}=6)$ & $3(50 \%)$ & $3(50 \%)$ \\
No prophylaxis $(\mathrm{n}=12)$ & 0 & $12(100 \%)$ \\
\hline
\end{tabular}

Notes: Patients were successfully discharged after SBP episode $(n=105)$. ${ }^{\mathrm{a} F r o m} 9 / 133$ patients undergoing liver transplantation in total, six patients underwent liver transplantation after study inclusion. 'Reasons for no prophylaxis in these patients were as follows: discharge to other hospital or rehabilitation clinic with ongoing intravenous antibiotic therapy $(n=3)$, transfer to palliative care $(n=3)$, recurring colitis from C. difficile $(n=I)$ and unidentifiable $(n=5)$.

Abbreviations: C. difficile, Clostridium difficile; SBP, spontaneous bacterial peritonitis; TIPS, transjugular intrahepatic portosystemic shunt. 


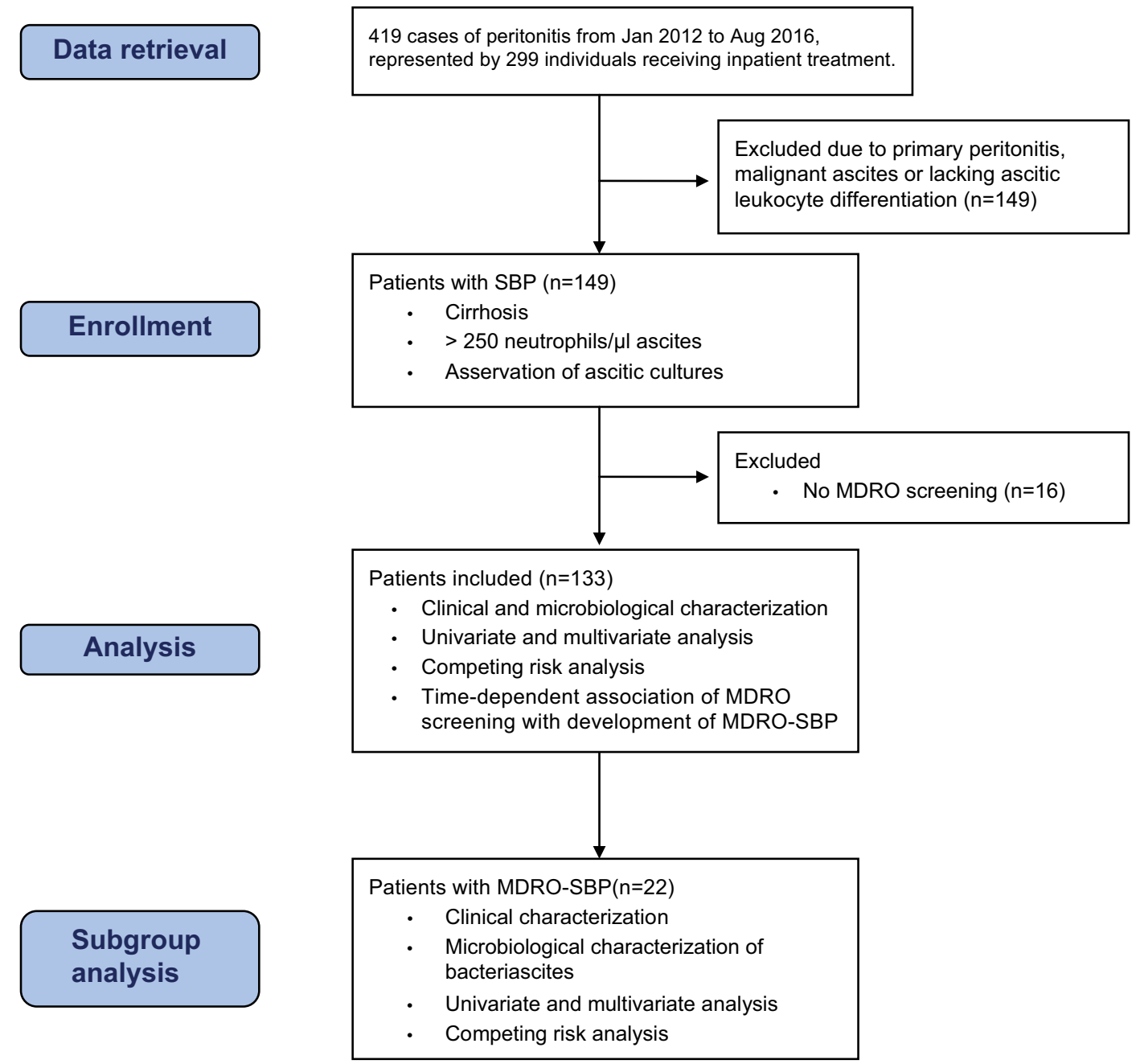

Figure SI CONSORT diagram of patients included in the study, analysis and subgroup analysis.

Notes: One patient who admitted with MDRO-SBP caused by E. coli expressing ESBL and QR did not undergo rectal MDRO swabs, since he had not been admitted to ICU/IMC and no regular MDRO screening was scheduled on normal wards. The patient was included due to ascitic MDRO detection and completion of cutaneous MDRO screening and was defined as MDRO negative in rectal screening. MDRO-SBP, SBP with evidence of MDROs or S. maltophilia in ascitic culture.

Abbreviations: E. coli, Escherichia coli; ESBL, extended-spectrum B-lactamase; ICU, intensive care unit; IMC, intermediate care unit; MDRO, multidrug-resistant organism; $\mathrm{QR}$, fluoroquinolone resistance; SBP, spontaneous bacterial peritonitis; S. maltophilia, Stenotrophomonas maltophilia.

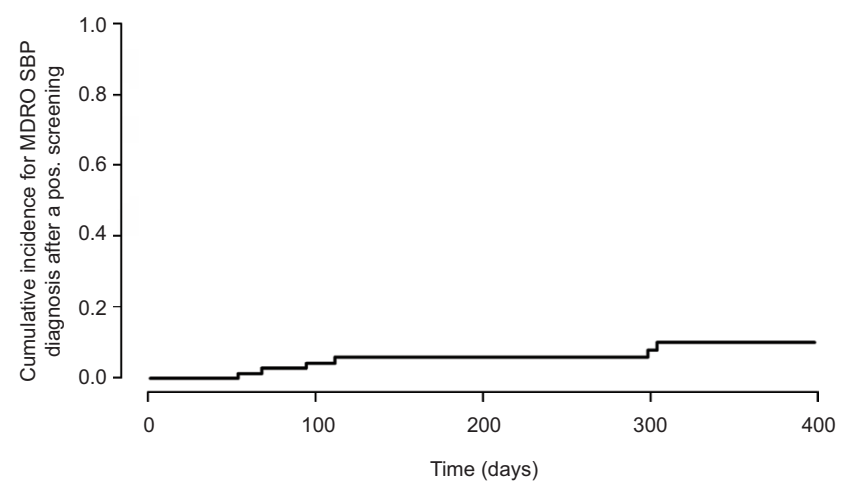

Figure S2 Cumulative incidence for MDRO-SBP diagnosis after a positive rectal SBP screening.

Note: MDRO-SBP, SBP with evidence of MDROs or S. maltophilia in ascitic culture. Abbreviations: MDRO, multidrug-resistant organism; SBP, spontaneous bacterial peritonitis; S. maltophilia, Stenotrophomonas maltophilia. 
Infection and Drug Resistance is an international, peer-reviewed openaccess journal that focuses on the optimal treatment of infection (bacterial, fungal and viral) and the development and institution of preventive strategies to minimize the development and spread of resistance. The journal is specifically concerned with the epidemiology of antibiotic resistance and the mechanisms of resistance development and diffusion in both hospitals and the community. The manuscript management system is completely online and includes a very quick and fair peerreview system, which is all easy to use. Visit http://www.dovepress.com/ testimonials.php to read real quotes from published authors.

Submit your manuscript here: https://www.dovepress.com/infection-and-drug-resistance-journal 